\title{
Simulating fracture network permeability in brown-coal slopes
}

\author{
R Hu Monash University, Australia \\ SDC Walsh Monash University, Australia \\ J Missen AGL Loy Yang Pty Ltd, Australia \\ N Anderson AGL Loy Yang Pty Ltd, Australia
}

\begin{abstract}
Slope stability of open cut brown-coal mines depends upon the permeability of the fracture network within the coal seam. In this paper, we provide a detailed analysis of the role of the fracture distribution on the permeability of brown-coal slopes and outline a model to capture this behaviour.

Brown coal is relatively impermeable, but heavily fractured. Hence, a stress-dependent fracture permeability model is employed that considers the spacing, orientation, aperture and frequency of the fractures to produce an effective permeability. We discuss how this model captures fracture heterogeneity and the effect of changing stress conditions on the fluid flow.

Fracture data collected from real mines exhibit significant degrees of heterogeneity, in particular, they may include a wide range of apertures and inter-fracture separations. The results of the effective permeability model demonstrate that in many cases slope permeability is dominated by highly permeable but low probability fractures. These results emphasise the need for models capable of capturing the effects of heterogeneity and uncertainty on the slope behaviour.
\end{abstract}

Keywords: lignite, fractured media, effective continuum models, numerical simulation

\section{Introduction}

The AGL Loy Yang open cut coal mine is located approximately $165 \mathrm{~km}$ southeast of Melbourne and provides almost 50\% of Victoria's energy requirements (AGL 2019). The AGL mining licence expires in 2037 and the mine is required to undergo progressive rehabilitation in the years leading up to that date (AGL 2019). Rehabilitation of the site entails cutting and filling benches of the mine no longer in production. To ensure these rehabilitated slopes are stable, it is necessary to understand the effect of water flow within the slopes, which are mostly comprised of brown coal (lignite), with layers of topsoil, clay and fill material. While solid brown-coal is relatively impermeable to water (e.g. Pan \& Connell 2012; Zhang et al. 2012), it is a highly brittle material containing numerous fractures that can open up and permit water flow into the slope. Excessive water retention within the slope may decrease the effective strength of the rock and lead to slope failure.

This paper presents an analysis of fracture distributions within brown-coal slopes, and introduces an effective continuum model to represent the slope's fracture network and its associated permeability. Fracture attributes such as spacing, aperture, and orientation are accounted for in the model, and their distributions are represented with probability density distribution functions. We compare the results from the model with other representations of the fracture network that only consider single values for each parameter and discuss the implications to the slope permeability.

\section{Model description}

In this section, we outline the numerical model used to represent the effective permeability of the brown-coal slopes. The model relies on probability density distribution functions to capture variations in 
fracture spacing, aperture and orientation. In the second part of this section, we describe the form of these distribution functions, and fit them to data obtained from a real fracture network. In the following section, we describe the implications of this model for the slope permeability.

\subsection{Effective permeability of fracture networks}

Solid brown-coal has a low permeability, approximately $2 \mathrm{mD}$ or $2 \times 10^{-12} \mathrm{~m}^{2}$ (Zhang et al. 2012). As such, the majority of the bulk permeability in the slope arises from the contribution of the in situ fracture network. To capture the effect of this fracture network, we introduce an effective continuum model based on the approach described in Chen et al. (2015).

The effective continuum model assumes that the permeability of the slope as a whole can be determined from the expected transmissibility of its in situ fracture network. Each fracture's permeability is determined from a parallel plate approximation:

$$
K=\frac{h^{2}}{12}
$$

where:

$K=$ the permeability of the fracture and $h$ is the hydraulic aperture (Witherspoon et al. 1980).

Perfectly smooth fractures do not exist in reality, and thus the permeability is affected by its roughness (i.e. the hydraulic aperture is not equal to the mechanical or observed aperture of the fracture), thus this estimate permeability should be viewed as an upper bound on the overall fracture permeability. Nevertheless, for fractures with large apertures (of the sort considered in this paper) the mechanical aperture and the hydraulic aperture are approximately equivalent (Barton et al. 1985). Assuming there exists a set of fractures separated by a spacing $d$, the three-dimensional permeability becomes:

$$
K_{i j}=\frac{h^{3}}{12 d}\left[\delta_{i j}-n_{i} n_{j}\right]
$$

where:

$\delta_{i j}=$ the Kronecker delta and $n_{i}$ is the fracture normal calculated from the dip and strike (Chen et al. 1999; Snow 1969).

Fractures open or close in response to the stresses acting upon them, in particular, changes in the fluid pressure within each fracture. A stress-dependent hydraulic aperture has been implemented to account for this behaviour in the present model. Several functional forms have been proposed to capture the aperture/stress relationship. For this paper, we adopt the model employed by Chen et al. (2015) and Seidle et al. (1992):

$$
h=h_{0} \exp \left(C \sigma_{e f f}\right)
$$

where:

$C=$ the fracture compressibility, $h_{0}$ is the initial hydraulic aperture and $\sigma_{\text {eff }}$ is the effective stress.

The fracture compressibility is a measure of the change in hydraulic of the fracture due to the stress applied. It can be expressed as:

$$
C=-\frac{1}{h} \frac{\Delta h}{\Delta \sigma_{e f f}}
$$

The compressibility is difficult to measure accurately, however an extensive literature review by Tan et al. (2019) reports a range of values up to $0.2 \mathrm{MPa}^{-1}$. In this paper, a middle value of $0.1 \mathrm{MPa}^{-1}$ is adopted. The effective stress is the stress acting perpendicular to the fracture less the pore pressure. 
Real fractures are not uniformly distributed, either in terms of their spacing, aperture or orientation. To capture this variability, the expression for the expected permeability of a single set of fractures given in Equation 2 is weighted by the probability of the fracture set occurring:

$$
E\left(K_{i j}\right)=\int K_{i j}(\boldsymbol{n}, h, d) \Phi(\boldsymbol{n}, h, d) d n d h d d
$$

where $\Phi(n, \boldsymbol{h}, \boldsymbol{d})$ gives the probability density for a particular fracture set with orientation $n$, hydraulic aperture $h$ and separation $d$. For the fracture set under consideration there is no clear correlation between the separation, aperture or orientation, and thus we assume that the probability density function for the fracture set is the product of the individual probability density distributions for the spacing, aperture and orientation e.g. $\Phi(n, \boldsymbol{h}, \boldsymbol{d})=\Phi_{n}(\boldsymbol{n}) \Phi_{h}(\boldsymbol{h}) \Phi_{d}(\boldsymbol{d})$. In the following section, we describe the form of this probability density distribution function and how it affects the permeability calculation.

\subsection{Fracture distribution models}

Many existing models such as Meng et al. (2011) and Adhikary \& Wilkins (2012) estimate the initial fracture permeability using either a median or expected value. However, fracture data from real slopes can vary over several orders of magnitude and, as a consequence may not be well modelled with a single value. Instead, to correctly represent the effect of the fracture network on the slope permeability, probability density distribution functions are introduced that capture the variations in the orientation, aperture and spacing.

Given a set of fracture data that includes the joint orientation, aperture and spacing; the stress-dependent permeability of the entire system can be estimated from the equations given in the previous section. As an example, data from the fractures in the Loy Yang coal mine are plotted on a stereonet (given in Figure 1). The fractures have a median dip of $78^{\circ}$ and dip direction of $85^{\circ}$. The fracture apertures have a mean value of $10.9 \mathrm{~mm}$ with a standard deviation of $15 \mathrm{~mm}$, while the fracture spacing has a mean of $3 \mathrm{~m}$ with a standard deviation of $4.8 \mathrm{~m}$.

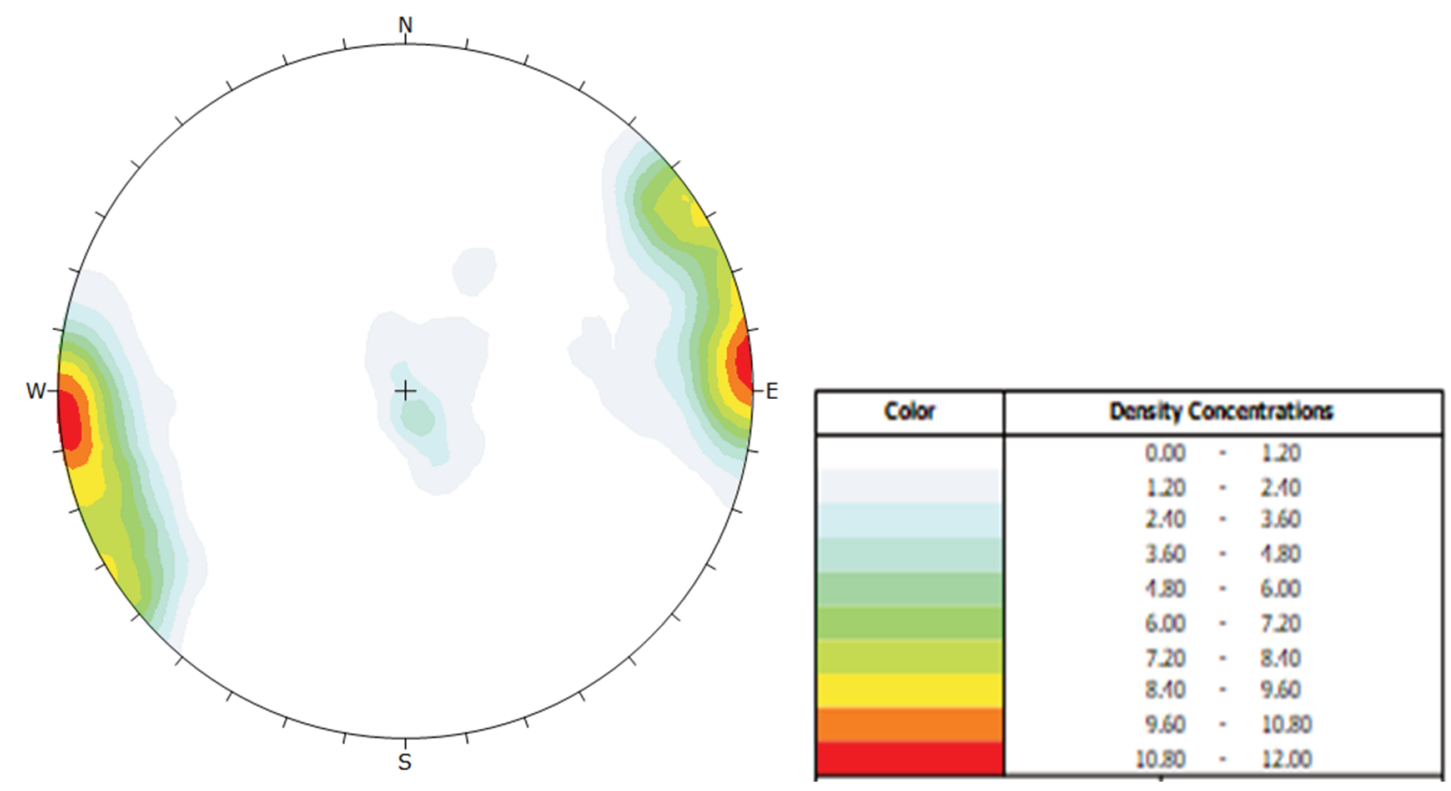

Figure 1 Stereonet of the fracture network (image created using the Dips software package)

While a majority of the fractures in the slope are vertically dipping, there are some horizontal bedding planes and joints. The fracture orientations are approximately normally distributed about three principal directions. We represent this variation using Gaussian functions fitted to the fracture density given in the stereonet. Correctly representing this variation is important to capture the anisotropy in the permeability tensor. However, the overall effect on the permeability is relatively minor (accounting for the variation in orientation around these directions changes the maximum permeability by approximately $10 \%$ ). As the effect of the fracture orientation on permeability tensor is relatively minor compared with the role of the effective stress, 
aperture and spacing, for the remainder of this paper we will treat the fracture orientations as if fixed along a single principle axis.

The slope batters at Loy Yang consists predominantly of brown coal with a density of $1.12 \mathrm{t} / \mathrm{m}^{3}$ (AGL 2017). Combining this with a maximum mine depth of approximately 220 m (AGL 2018), the resultant effective vertical stress applied is approximately $2 \mathrm{MPa}$. This assumes no water is present so there is no additional weight or pore pressure. The resultant effective permeabilities with different stresses applied are shown in Table 1.

Table 1 Mean permeabilities calculated for the fracture network for different effective stresses

\begin{tabular}{|c|c|c|c|c|c|c|}
\hline \multirow{3}{*}{$\boldsymbol{\sigma}_{\mathrm{eff}}$} & \multicolumn{6}{|c|}{ Mean permeability of the fracture sets } \\
\hline & \multicolumn{2}{|c|}{$0 \mathrm{MPa}$} & \multicolumn{2}{|c|}{$1 \mathrm{MPa}$} & \multicolumn{2}{|c|}{$2 \mathrm{MPa}$} \\
\hline & Mean & $\begin{array}{l}\text { Std. } \\
\text { deviation }\end{array}$ & Mean & $\begin{array}{l}\text { Std. } \\
\text { deviation }\end{array}$ & Mean & $\begin{array}{l}\text { Std. } \\
\text { deviation }\end{array}$ \\
\hline Raw data & $8.4 \times 10^{-6} \mathrm{~m}^{2}$ & $1.1 \times 10^{-4} \mathrm{~m}^{2}$ & $6.2 \times 10^{-6} \mathrm{~m}^{2}$ & $8.1 \times 10^{-5} \mathrm{~m}^{2}$ & $4.6 \times 10^{-6} \mathrm{~m}^{2}$ & $6.0 \times 10^{-5} \mathrm{~m}^{2}$ \\
\hline $\begin{array}{l}\text { Calculated from } \\
\text { Mean values }\end{array}$ & $4.8 \times 10^{-8} \mathrm{~m}^{2}$ & - & $3.6 \times 10^{-8} \mathrm{~m}^{2}$ & - & $2.6 \times 10^{-8} \mathrm{~m}^{2}$ & - \\
\hline $\begin{array}{l}\text { Log-normal } \\
\text { distribution }\end{array}$ & $1.4 \times 10^{-5} \mathrm{~m}^{2}$ & $3.8 \times 10^{-4} \mathrm{~m}^{2}$ & $1.0 \times 10^{-5} \mathrm{~m}^{2}$ & $2.8 \times 10^{-4} \mathrm{~m}^{2}$ & $7.6 \times 10^{-6} \mathrm{~m}^{2}$ & $2.1 \times 10^{-4} \mathrm{~m}^{2}$ \\
\hline $\begin{array}{l}\text { Log-beta } \\
\text { distribution }\end{array}$ & $2.0 \times 10^{-6} \mathrm{~m}^{2}$ & $2.5 \times 10^{-5} \mathrm{~m}^{2}$ & $1.5 \times 10^{-6} \mathrm{~m}^{2}$ & $1.9 \times 10^{-5} \mathrm{~m}^{2}$ & $1.1 \times 10^{-6} \mathrm{~m}^{2}$ & $1.4 \times 10^{-5} \mathrm{~m}^{2}$ \\
\hline
\end{tabular}

The fracture data provided by the AGL Loy Yang Coal Mine contained apertures up to $300 \mathrm{~mm}$ and separations up to $25 \mathrm{~m}$. The data also contains outliers of $1,000 \mathrm{~mm}$ fracture apertures and $1,000 \mathrm{~m}$ separations. However, these were considered to be missing data where a proper measurement could not be taken and were instead excluded from the analysis.

To investigate the effect of the variation in the aperture and spacing in the model, the fracture data was fitted to both log-normal and log-beta probability distributions. These methods were used to determine an expected permeability for the slope and can be compared with the results from the fracture set. The probabilistic fits of the data also provide a likely range of permeability so the uncertainty may be quantified.

The separations and apertures of the Loy Yang batter fractures are approximately log-normally distributed, similar to the reported distributions for other fracture sets (Renshaw 1995). The log-normal distribution was fitted by calculating the cumulative probability for each spacing and minimising the combined difference with the cumulative probability from the original dataset. For a log-normal distribution, the fitted cumulative distribution function (CDF) is given by:

$$
C D F(x)=\frac{1}{2}\left[1+\operatorname{erf}\left(\frac{\ln (x)-\mu}{\sqrt{2} \sigma}\right)\right]
$$

where:

$$
\begin{array}{lll}
\operatorname{CDF}(x)= & \text { the cumulative distribution function of } x . \\
\text { erf } & = & \text { the error function, } \mu \text { is the log-mean. } \\
\sigma & = & \text { the log-standard deviation. }
\end{array}
$$

Log-normal distributions were fitted to both the fracture aperture and the inter-fracture separation (i.e. the distance between each fracture minus the fracture aperture). By fitting the inter-fracture separation, rather than the fracture separation, ensures that the fracture aperture is never greater than the separation between fractures. 
Nevertheless, while the standard log-normal distribution provides a good fit to the fracture data, the support of distribution is unbounded for large values of each variable, i.e. there is a very small but finite probability for fractures of any aperture and separation - no matter how large. As this long tail may skew the data (i.e. extremely low probability events may have an undue influence on the results), the data was also fitted with a log-beta distribution to test the effect of the form of the distribution on the final result. An attempt was also made to fit both distributions using the standard beta distribution function. However, the fit was an unsatisfactory match for both the spacing and aperture due to the wide range of values and sharp peak at small values in both datasets.

The log-beta function is similar to a log-normal density distribution function in that it fits a probability distribution to the natural log of the underlying data. As the name implies, however, the beta distribution is used in place of the normal distribution. The standard beta distribution is scaled between 0 and 1 , and thus a choice must be made for the range of values for the log-beta function. For the purpose of this paper, the range of the log-beta function was set to three log-standard deviations on either side of the log-mean of the data (i.e. $\mu-3 \sigma<\log (x)<\mu+3 \sigma$ ). This produces a distribution that captures the bulk of the data, but eliminates the effect of the long tail. The CDF of the log-beta function is given by:

$$
C D F(x)=\frac{\Gamma(\alpha+\beta)}{\Gamma(\alpha) \Gamma(\beta)} \int_{t=0}^{t_{\max }} t^{\alpha-1}(1-t)^{\beta-1} d t
$$

where the limit of the integral is given by $t_{\max }=(\log (x)-\mu+3 \sigma) / 6 \sigma$ and $\Gamma(x)$ is the gamma function. The two shape parameters $(\alpha$ and $\beta$ ) for the log-beta distributions were found by minimising the misfit between the raw CDF from the data and that given by the log-beta function. This yielded an $\alpha$ and $\beta$ of 13.9 and 17.1 for the fracture spacing and 8.8 and 10.9 for the fracture aperture.

The CDFs for the raw data for both the aperture and spacing, and the fitted log-normal and log-beta functions are shown in Figure 2 by plotting the log of the aperture and spacing versus the associated CDF. As is evident from the plots both the standard log-normal distribution and the log-beta distributions have excellent agreement with both datasets for most of the range of apertures and separations.

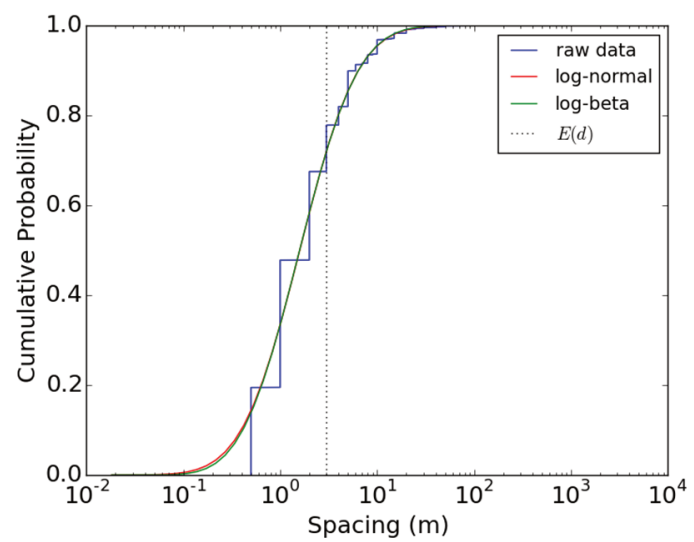

(a)

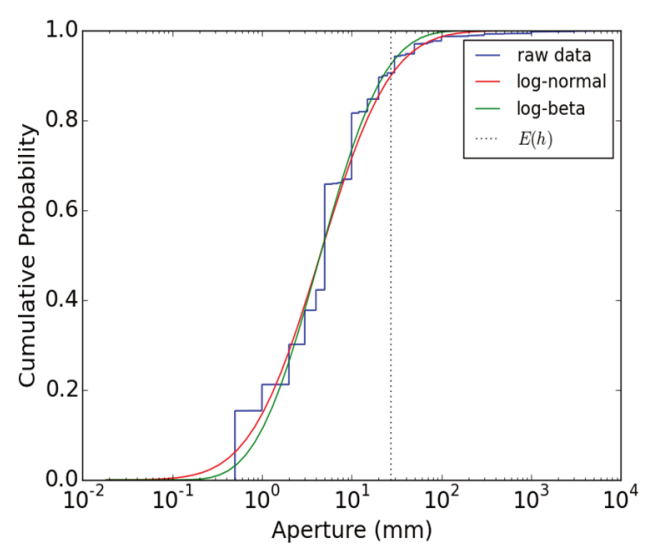

(b)

Figure 2 Raw and fitted probability distribution functions for a) the fracture spacing and b) the fracture aperture

\section{$3 \quad$ Fracture permeability}

The average or expected permeability of the fractures, $E(K)$, in the raw data is $8.5 \times 10^{-6} \mathrm{~m}^{2}$. This is over two orders of magnitude greater than the permeability calculated if only the mean spacing and aperture are used (i.e. $E(h)^{3} / 12 E(d)=4.8 \times 10^{-8} \mathrm{~m}^{2}$ ). In comparison, the expected permeability using the log-normal fits was determined to be $1.4 \times 10^{-5} \mathrm{~m}^{2}$ while the expected permeability using the log-beta distribution was found to be $2.0 \times 10^{-6} \mathrm{~m}^{2}$. 
Figure 3 plots the relative contribution to the expected permeability from each combination of aperture and spacing for both the log-normal and log-beta distributions. In both cases, the dominant contribution to the permeability arises from small values of the fracture separation (less than one metre) with comparatively large apertures (between 10-20 cm). While fractures in this range are very rare, the cubic dependence on the aperture exaggerates their influence on the mean permeability. The unbounded log-normal distribution overestimates the permeability, while the beta distribution gives an underestimate as it gives a lower probability to some of the larger apertures in the dataset. Nevertheless, both probability distributions yield similar permeability distributions to that given by the raw fracture set (Figure 4).

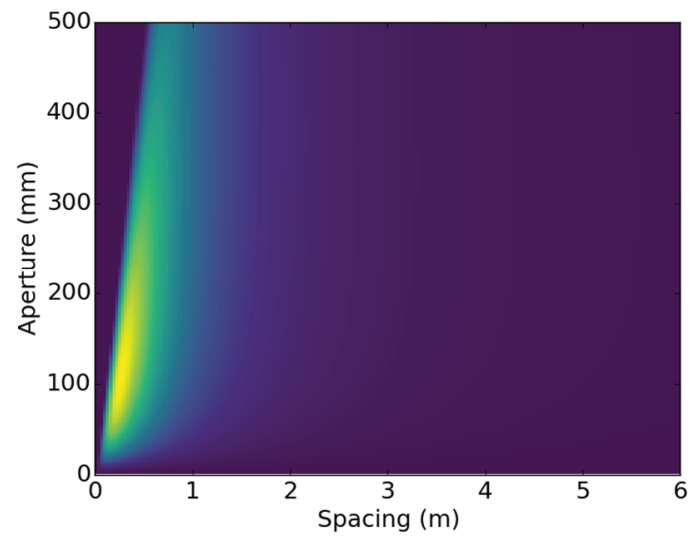

(a)

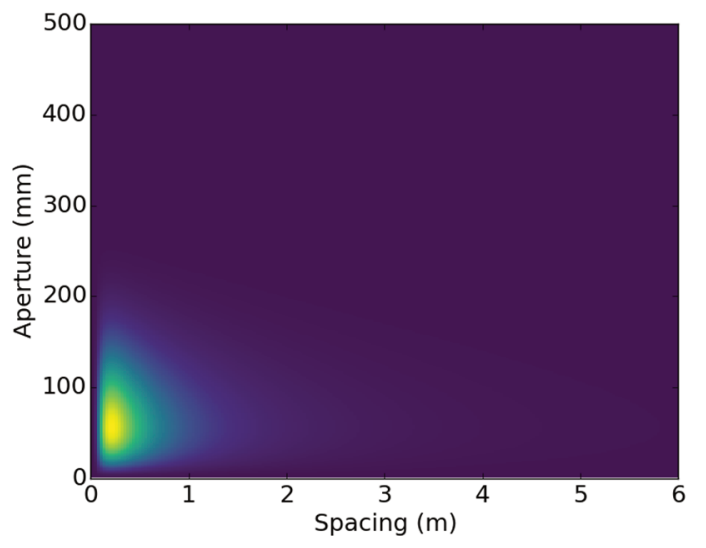

(b)

Figure 3 Relative contributions to the permeability for a) the log-normal and b) log-beta probability distributions

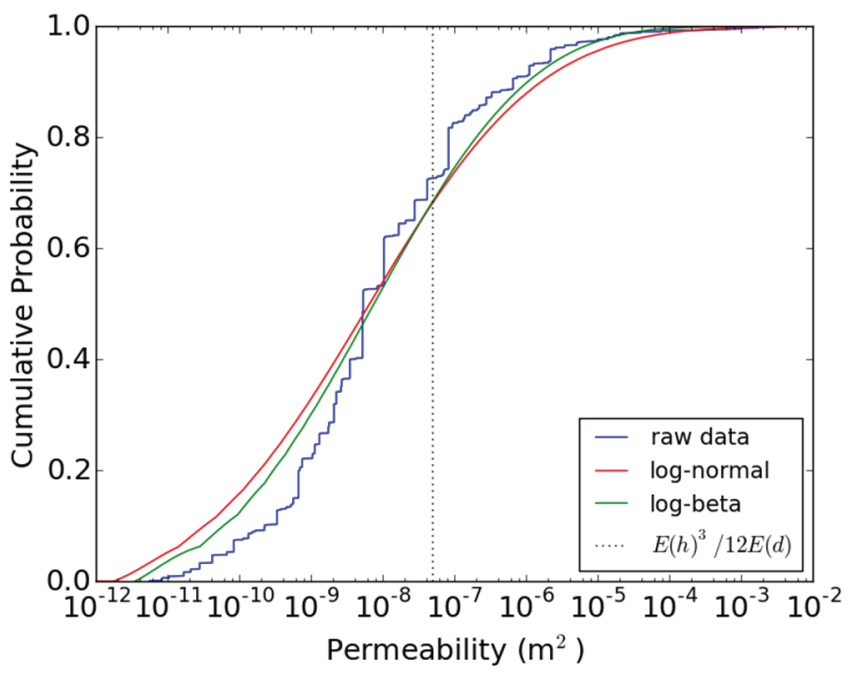

Figure 4 Semilog plot of the cumulative distribution function of the (log) permeability for the raw data (blue), log-normal (red), and log-beta (green). The dashed line indicates the permeability estimate calculated from the average fracture aperture and spacing

A difficulty in making a direct comparison between the mean permeabilities of the probability distributions and that of the raw data from the slope itself, is that the average is sensitive to the sample size due to the influence of infrequent fractures with large permeabilities. In Figure 5, we compare the mean fracture permeability given by the raw data with fracture permeabilities given by random samples of the same size from the fitted probability density distributions. The average permeability calculated from the mean values is also included for reference. The permeabilities of both sub-samples is generally lower than that for the distributions, again because they tend not to sample the high-permeability but low probability fractures. For this reason, the log-beta function, which provides an arguably better fit to the raw fracture distribution 
(as illustrated in Figure 4), fails to match the permeability of the raw dataset for any of the sample realisations. Conversely, while the entire log-normal distribution predicts a higher expected permeability, the observed expected fracture permeability is within the range of values predicted if a finite sample is considered. In fact, approximately $30 \%$ of the time the model produced a fracture set with a lower average permeability than that in the Loy Yang dataset.

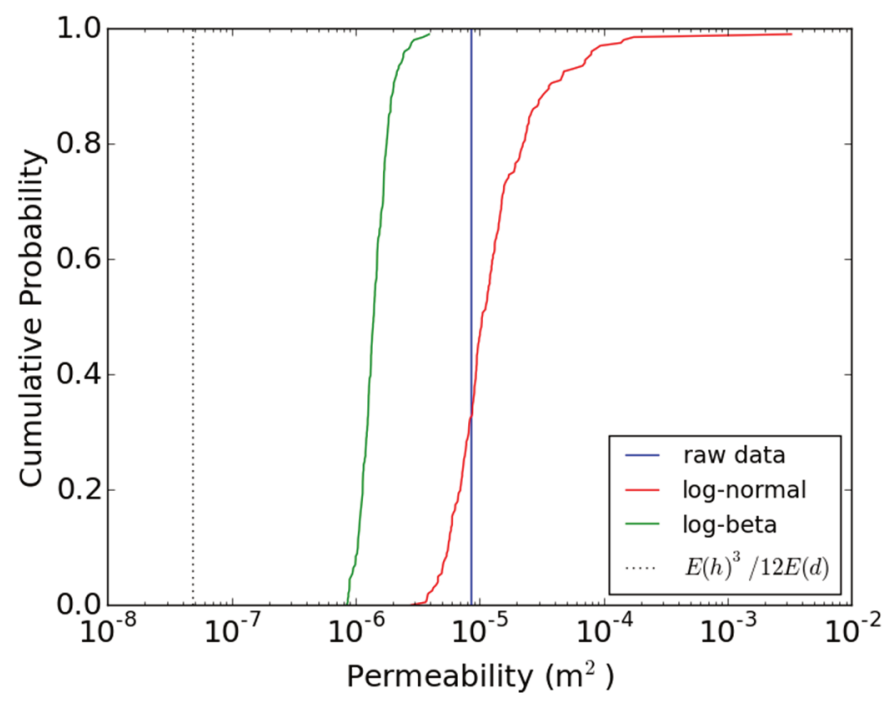

Figure 5 Semilog plots comparing the distribution of mean permeabilities of random samples with the same size as the raw data. The plots show the range of permeabilities calculated from log-normal (red) and log-beta (green) distributions with that of the raw data

By fitting to the sample data, the beta function fails to properly match the probability of larger aperture fractures, which skew the permeability calculation. A solution to this problem is to fit the beta function to the log-normal aperture distribution, rather than the raw data. The advantage of this approach over using the log-normal distribution directly, is that the beta function is able to capture the high-end of the probability distribution while also eliminating the possibility of excessively large fracture apertures. Figure 6 shows the effect of replacing the log-beta fitted to the raw data with that fitted to the log-normal data on the sub-sampled permeability estimates. The result produces a sub-sample distribution that, like the original log-normal distribution, includes the observed expected permeability, but does so without spuriously large fracture apertures.

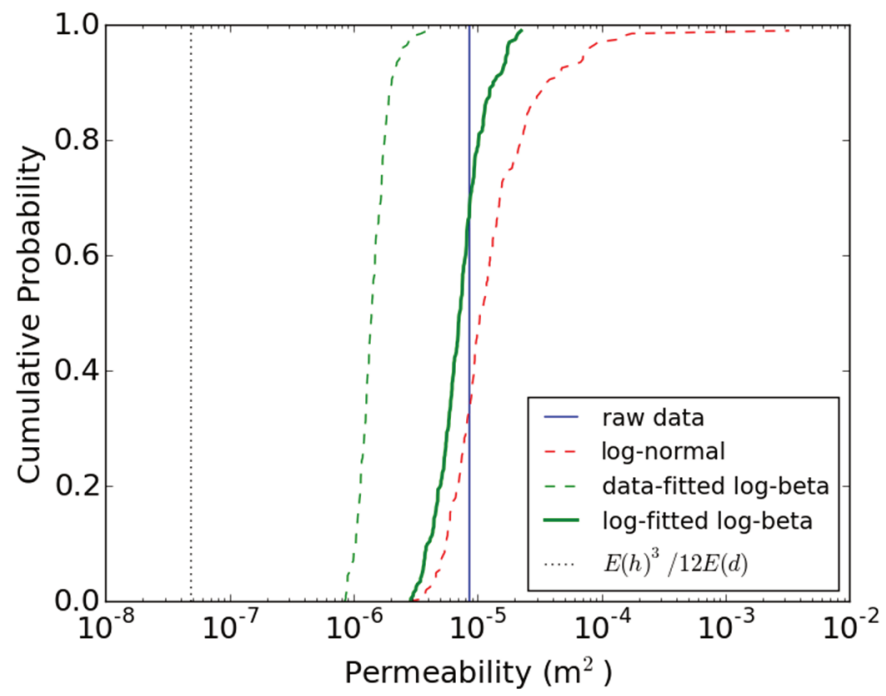

Figure 6 Semilog plots repeating Figure 5, but showing the effect of using the log-beta function fitted to the log-normal distribution (solid green line) on the sub-sampled permeability distribution 


\section{Conclusion}

As solid brown-coal is relatively impermeable, the bulk permeability of brown-coal slopes is largely dictated by their internal fracture network. Correctly characterising the permeability of this network is crucial to predict the long-term behaviour of this material, particularly when considering the stability of open cut coal mines.

In this paper, we consider an effective fracture network model describing the bulk permeability of brown coal. The model uses a parallel plate approximation to capture the permeability's dependence on the fracture orientation, spacing and aperture, as well as variations in these parameters and the effects of stress upon the fracture network.

The greatest influence on the overall fracture permeability stems from the heterogeneity in the fracture network itself. In particular, the network permeability is strongly influenced by small numbers of fractures with small separations and relatively large apertures. In particular, this implies that it is insufficient to rely on the average spacing and aperture (or any other single value) to calculate the overall permeability of the slope.

Instead, probability density distribution functions were introduced to represent the variation in fracture aperture and separation. Two distinct classes of probability distribution models were considered, log-normal and log-beta functions, to study the effect of the form of these functions on the predicted fracture permeability. Both functions provided a significantly better match to the average permeabilities in the fracture network than using single values for the aperture and spacing, and provided a reasonable approximation of the permeability distribution.

The results showed that the mean permeability is sensitive to the sample size, due to the dominant influence of a small number of highly permeable but very infrequent fractures. As a result, a direct comparison between the expected permeability from the fracture data distributions, may be misleading. To overcome this bias, we instead considered the expected permeability of random samples of fractures from both permeability distributions, and compare their range of permeabilities with that predicted by the slope data. The wide variation in predicted permeabilities highlights the need for numerical models that account for fracture heterogeneity. The analysis revealed that the log-normal distribution provides a more likely match to the actual data. However, it is also possible to reproduce the observed expected permeability by introducing a beta function fitted to the log distribution, rather than the original data. This approach provided a match that is similar in accuracy to the original log-normal distribution, but eliminates predictions of spuriously large fracture permeabilities.

\section{Acknowledgement}

We would like to thank AGL Loy Yang for the necessary funding and data for this study.

\section{References}

Adhikary, DP \& Wilkins, A 2012, Reducing the Impact of Longwall Extraction on Groundwater Systems, CSIRO, Collingwood.

AGL 2017, Ground Control Management Plan, Revision 03, internal report.

AGL 2018, 'AGL Loy Yang Mine Sustainability Report 2017-2018', AGL, https://www.agl.com.au/-/media/aglmedia/documents/aboutagl/how-we-source-energy/loy-yang/sustainability-report-17-18-20180815.pdf?la=en\&hash=8A06AE15DE05BDF426A6A8 2E16E4BF8E

AGL 2019, AGL Loy Yang Power Station, viewed 9 October 2019, https://www.agl.com.au/about-agl/how-we-source-energy/loyyang-power-station

Barton, N, Bandis, S \& Bakhtar, K 1985, 'Strength, deformation and conductivity coupling of rock joints', International Journal of Rock Mechanics and Mining Sciences \& Geomechanics Abstracts, vol. 22, no. 3, pp. 121-140.

Chen, M, Bai, M \& Roegiers, JC 1999, 'Permeability tensors of anisotropic fracture networks', Mathematical Geology, vol. 31, no. 4, pp. 335-373.

Chen, D, Pan, Z \& Ye, Z 2015, 'Dependence of gas shale fracture permeability on effective stress and reservoir pressure: Model match and insights', Fuel, vol. 139, pp. 383-392.

Meng, ZP, Zhang, JC \& Wang, R 2011, 'In situ stress, pore pressure and stress-dependent permeability in the Southern Qinshui Basin', International Journal of Rock Mechanics \& Mining Sciences, vol. 48, pp. 122-131. 
Pan, Z \& Connell, LD 2012, 'Modelling permeability for coal reservoirs: A review of analytical models and testing data', International Journal of Coal Geology, vol. 92, pp. 1-44.

Renshaw, CE 1995, 'On the relationship between mechanical and hydraulic apertures in rough-walled fractures', Journal of Geophysical Research, vol. 100, no. B12, pp. 24629-24636.

Seidle, JP, Jeansonne, DJ \& Erickson, DJ 1992, 'Application of matchstick geometry to stress dependent permeability in coals', Proceedings of the Rocky Mountain Regional Meeting 1992, Society of Petroleum Engineers, Inc., Richardson, pp. 433-444.

Snow, DT 1969, 'Anisotropic permeability of fractured media', Water Resources Research, vol. 5, no. 6, pp. 1273-1289.

Tan, Y, Pan, Z, Feng, X, Zhang, D, Connell, LD \& Li, S 2019, 'Laboratory characterisation of fracture compressibility for coal and shale gas reservoir rocks: A review', International Journal of Coal Geology, vol. 204, pp. 1-17.

Zhang, L, Aziz, N, Ren, T, Nemcik, J \& Wang, Z 2012, 'Permeability testing of coal under different triaxial conditions', Proceedings of the 12th Coal Operators' Conference, The Australasian Institute of Mining and Metallurgy, Melbourne, pp. 277-285.

Witherspoon, PA, Wang, JSY, Iwai, K \& Gale, JE 1980, 'Validity of Cubic Law for fluid flow in a deformable rock fracture', Water Resources Research, vol. 16, no. 6, pp. 1016-1024. 
